\title{
MANEJO CLÍNICO DE LA DIMENSIÓN VERTICAL CON PRÓTESIS FIJA Y PRÓTESIS REMOVIBLE CON EJE ROTACIONAL DE INSERCIÓN
}

\section{CLINICAL MANAGEMENT OF THE VERTICAL DIMENSION WITH FIXED PROSTHESIS AND REMOVABLE PARTIAL DENTURE, WITH ROTATIONAL INSERTION AXIS}

\author{
Elías Pizarro N ${ }^{1 a, b}$, Gómez Stella L ${ }^{1 a, c}$ Muñante Arzapalo K ${ }^{1 a, d}$
}

\section{RESUMEN}

La alteración de la dimensión vertical puede ser ocasionada por diferentes factores, dentro de los cuales, encontramos pérdida parcial de piezas dentarias, desgastes patológicos de las superficies oclusales que son provocados por distintas etiologías, siendo uno de los más comunes el bruxismo. Esto trae como consecuencia alteraciones a nivel del sistema estomatognático que provocan secuelas estéticas y funcionales. Es por ello la importancia del conocimiento teórico y clínico del edentulismo parcial cuando la dimensión vertical se encuentra alterada, pues como sabemos existen en la actualidad muchos biomateriales y técnicas para rehabilitar un paciente con estas características, sin embargo, todo parte de un correcto diagnóstico. Tradicionalmente se han usado diferentes métodos para establecer la dimensión vertical adecuada, sin embargo, aún no hay un método único capaz de brindar una total exactitud. El objetivo de este trabajo es poder compartir el abordaje a un paciente con alteración de la dimensión vertical a través de la confección de prótesis fija y prótesis parcial removible (PPR) con doble eje de inserción. Datos principales se realizó la rehabilitación completa a un paciente de 82 años edéntulo parcial superior que presentaba disminución de la dimensión vertical por presentar habito parafuncional (bruxismo céntrico y excéntrico). Principales comentarios: Los resultados fueron satisfactorios para el paciente ya que se logró la rehabilitación completa a través de prótesis combinada (fija y removible) restableciendo así la dimensión vertical y por lo tanto teniendo resultados funcionales y estéticos. Conclusiones: El uso de prótesis fija y Prótesis removible con eje rotacional de inserción es una alternativa de tratamiento que permite devolver de forma óptima las funciones de la cavidad oral dando como resultado el confort del paciente.

Palabras claves: Dimensión vertical, Prótesis fija; prótesis parcial removible (Fuente: DeCS BIREME)

\section{ABSTRACT}

The alteration of the vertical dimension can be caused by different factors, among which, we find partial loss of dental pieces, pathological wear of the occlusal surfaces that are caused by different etiologies, being one of the most common bruxism. This results in alterations in the stomatognathic system that causes aesthetic and functional sequelae. That is why the importance of theoretical and clinical knowledge of partial edentulism when the vertical dimension is altered, because as we know there are currently many biomaterials and techniques to rehabilitate a patient with these characteristics, however, all part of a correct diagnosis.

Traditionally different methods have been used to establish the appropriate vertical dimension; however, there is not yet a single method capable of providing total accuracy. The objective of this work is to share the approach to a patient with alteration of the vertical dimension through the manufacture of fixed prosthesis and PPR with double insertion axis. Main data a complete 82 -yearold edentulous patient who had a decrease in the vertical dimension due to parafunctional habit (centric and eccentric bruxism) was completely rehabilitated. Main comments: The results were satisfactory for the patient since full rehabilitation was achi eved through combined prosthesis (fixed and removable) thus restoring the vertical dimension and therefore having functional and aesthetic results. Conclusions: The use of fixed prosthesis and PPR double axis of insertion is an alternative of treatment that allows returning of optimal form the functions of the oral cavity giving like result the comfort of the patient.

Keywords: Vertical dimension, Dentadure, partial, Fixed; Dentadure, partial removable. (Source: MeSH NLM)

Recibido: 23 de abril de 2018

Aprobado: 12 de agosto de 2018

Publicado: 30 de setiembre de 2018

${ }^{1}$ Universidad Inca Garcilaso de la Vega

${ }^{a}$ Cirujano Dentista, ${ }^{b}$ Residente de Rehabilitación Oral

${ }^{\mathrm{c}}$ Coordinador de la especialidad de Rehabilitación Oral

dMagister en Estomatología, Docente

Correspondencia:

Katty Muñante Arzapalo

Correo electrónico: khatycita@gmail.com
Este es un artículo Open Access distribuido bajo la licencia Creative Commons Atribución-NoComercial- Compartir Igual 4.0

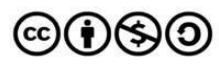

Citar como: López Gallegos E, Armas A, López Ríos E. Nuñez A, Tatés Almeida K. Prevalencia de hábitos deletéreos y maloclusiones en dentición mixta en niños de la ciudad de Quito, Ecuador. KIRU. 2018; 15(3): 140 - 145. https://doi.org/10.24265/kiru.2018.v15n3.06 


\section{INTRODUCCIÓN}

Cuando se realiza una revisión de la definición de la dimensión vertical encontraremos diferentes conceptos que van de lo teórico a lo clínico. Según Dawson, la dimensión vertical es la posición de relación estable entre el maxilar y mandíbula cuando hay máxima intercuspidación, donde el determinante de la dimensión vertical (DV) son los músculos, en base a su longitud repetitiva de contracción, indica que el patrón de cierre es extremadamente constante. ${ }^{(1)}$

En el atlas de análisis Oclusal, se define a la dimensión vertical oclusal (DVO) como una medida de la dimensión facial tomada verticalmente, con los dientes, rodetes de cera, dentaduras completas u otras restauraciones en oclusión céntrica. De la misma manera podemos citar a muchos autores, pero casi todas coinciden en que la DV es la medida de la altura del tercio inferior de la cara; siendo ésta una relación maxilomandibular vertical. ${ }^{(2)}$

Al realizar la planificación en las rehabilitaciones completas con prótesis fija o combinada con aparato removibles, cuya causa haya sido por edentulismo parcial y/o degaste generalizado, la estabilización oclusal es el motivo primordial. Sin embargo, en algunos casos se necesitan variaciones en la dimensión vertical de oclusión.

Frecuentemente el diagnóstico que nos indica realizar modificaciones en la DVO es el grado de desgaste de las superficies dentarias, que puede estar acompañados con signos faciales como disminución de la altura del tercio inferior de la cara, pseudoprognatismo, colapso labial y/o aparición de arrugas faciales acentuándose en los pliegues geníanos y labiomentonianos; otras de las manifestaciones usualmente encontradas podemos mencionar la falta de espacio protésico siendo esta una de las complicaciones más relevantes.

Para determinar la dimensión vertical existe un sin número de alternativas escritas en la literatura (métodos fonéticos, estéticos, antropométricos, cefalométricos, con instrumentos, etc). Sin embargo, hasta la fecha no existe un método único capaz de responder con total exactitud. Es por eso que se recomienda el uso de varios métodos para tener la certeza que se tiene una adecuada medida para realizar la rehabilitación.

Los requisitos que debe tener una dimensión vertical de oclusión correcta :

- Debe consentir una distancia interoclusal adecuada entre la posición de descanso y la oclusión céntrica es decir, permitir que se exprese el espacio libre, el cual se puede determinar sustrayendo la medición de la dimensión vertical en oclusión de la dimensión vertical en reposo.

- Una longitud de dientes y una altura de cúspides mecánicamente saludables, estéticamente buena y fonéticamente correcta.

- Podemos comprobar también la atura correcta de la dimensión vertical utilizando datos referidos por el paciente, dentro de estos encontramos: la comodidad por la utilización de las prótesis provisionales que pueden ser PPR acrílicas, coronas entre otros. Ausencia de síntomas que nos indican una alteración a nivel muscular producto de un aumento excesivo de la DV, así como también el confort del nuevo perfil facial.

\section{DESCRIPCIÓN DEL CASO CLÍNICO}

Paciente masculino, 82 años de edad en buen estado general y sin antecedentes patológicos relevantes, acude a consulta odontológica refiriendo como motivo de consulta, sensibilidad dentaria al frio y calor acompañado de dificultad masticatoria. Al realizar la exploración extraoral encontramos:

Desproporción de los tercios faciales, siendo más evidente en el tercio inferior mediante la manifestación de las comisuras labiales abatidas, el surco nasogeniano más pronunciado y las líneas de expresión facial en general más pronunciadas.(fig 1 a,b,c).

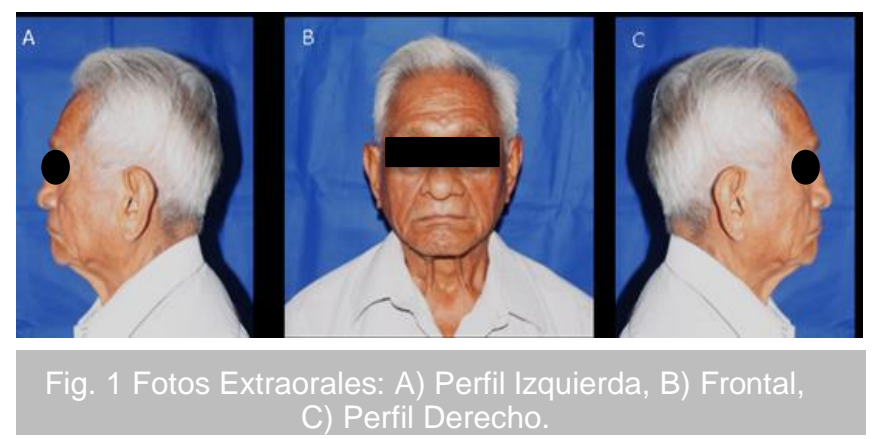

Al examen intraoral presentaba edéntulismo parcial, forma de arcada cuadrangular para ambos maxilares, Encía marginal: a nivel de piezas $11 ; 12,13 ; 21 ; 22$; 23,33/34; 46 inflamadas. en el maxilar superior: lesiones cariosas: 14 ; coronas provisionales: 12 ; 22 , Coronas metal cerámicas piezas. 11,15; 17; 23,27 en mal estado, pernos metálicos: 12; 15; 23, Desgastes dentarios: 13 ; $21\left(4^{\circ}\right)$.

En el maxilar inferior: Restauraciones en las piezas 35,36; 45; coronas metal cerámicas pieza. 42; pernos metálicos piezas.12;15; 23 endodoncias pieza 31; todo en mal estado. 
Desgastes dentarios: Grado 2: 34;35;36;44;45;46; Grado 3: 33;36;38; Grado 4: 31,32;41;42;43. (Eigura 2 A, B, C), (Figura 3 A.B, C).

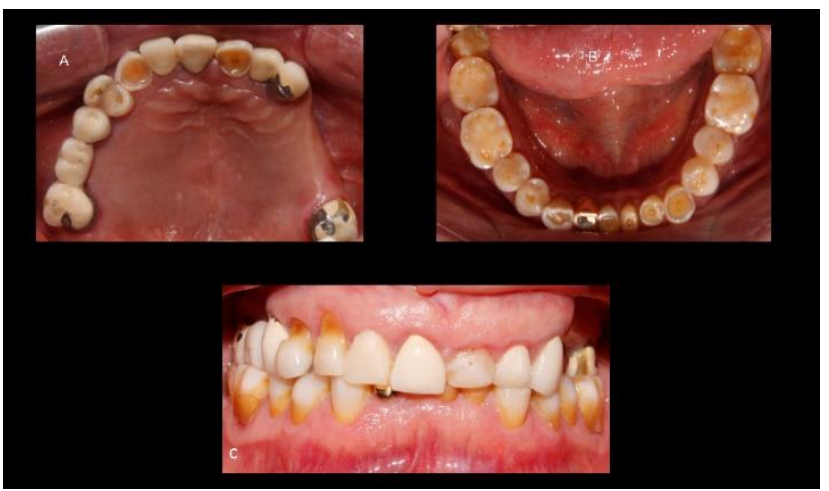

Figura 2. Fotos Intraorales: oclusales y de frente

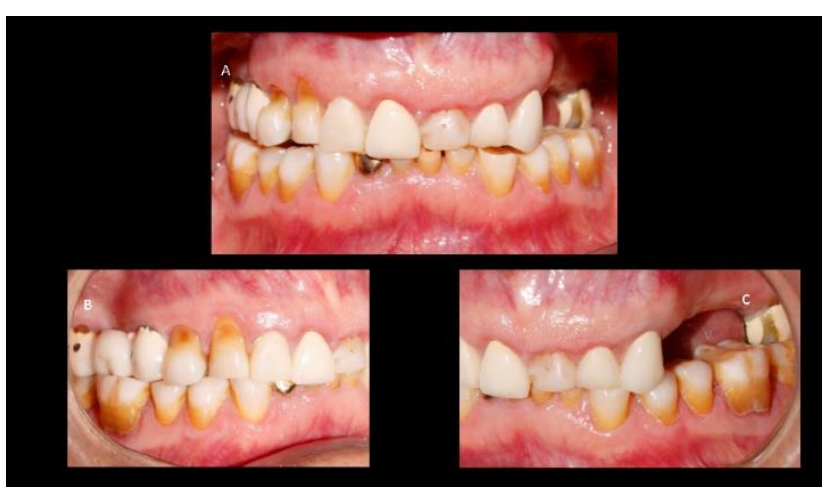

\section{Figura 3. Fotos Intraorales: Frontal, Derecha e} Izquierda

Con respecto al registro de la dimensión vertical (DVPDVO), se encontró que el espacio libre (EL) era muy amplio (entre 7 y $8 \mathrm{~mm}$,) demostrando la pérdida de DVO.

Al realizar los exámenes complementarios: radiografía panorámica, periapicales, modelos de estudio y encerado de diagnóstico articulados con toma de registro RIM Y RAF (Figura 4 a, b, c) ;(Figura 5 a, b, c) y las respectivas interconsultas se logró establecer el diagnóstico.

\section{DIAGNOSTICO DE LA OCLUSION:}

\section{Desorden funcional oclusal}

Disarmonía oclusal

- Alteración de la guía anterior

- Alteración del plano Oclusal

- Interferencias en céntrica, lateralidades y protusiva

- Edéntulo parcial superior
Parafunción:

Bruxismo céntrico y excéntrico

Después de establecer el diagnóstico, se le presenta al paciente y a sus familiares distintas posibilidades de abordar el caso clínico, quienes optan por el tratamiento combinado de prótesis fija y PPR con doble eje de inserción siendo así establecida la programación de las citas para cumplir con el objetivo.

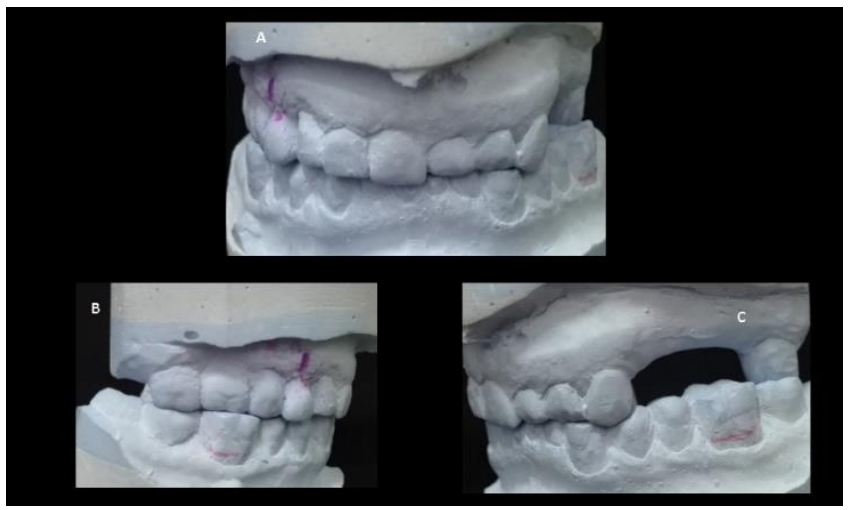

Figura 4. Fotos Intraorales: Frontal, Derecha e Izquierda

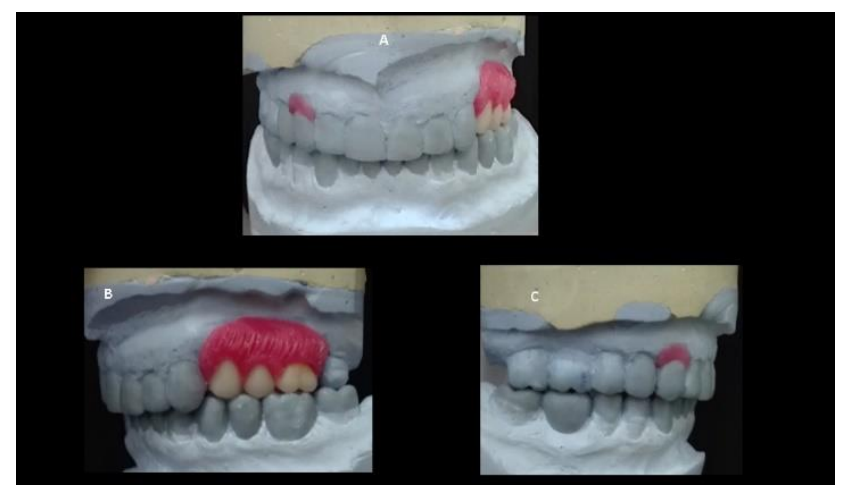

Figura 5. Encerado diagnostico

El paciente presentaba además gingivitis, periodontitis localizada y recesión gingival generalizada.

En tejidos duros presentaba lesiones dentales no cariosas como abfracción y atrición, pulpitis irreversible asintomática en pieza 21; Necrosis pulpar piezas 41 ; 42. También mostraba una periodontitis apical asintomática en la pieza 31 .

\section{PLAN DE TRATAMIENTO}

\section{Fase Sistémica}

Controles en gastroenterología 


\section{Fase de higiene y correctiva}

- Servicio de periodoncia: fisioterapia oral

Gingivoplastia y gingivectomia de la 13 a la 23.

(Fig.6a)

- Servicio de Endodoncia:

Endodoncia: Pzas. 21; 42;41;46

Retratamiento endodóntico: Pzas. 12; 23; 15; 31

\section{FASE REHABILITADORA}

\section{Fase estabilizadora Maxilar superior}

$\checkmark \quad$ Retiro de coronas metálicas, y provisionales Pzas 11;12;22;15;17;23;27

$\checkmark$ Retiro de postes colados: pzas 12;15;23

$\checkmark$ Tallado y coronas provisionales pzas. $11 ; 12 ; 13 ; 14 ; 21 ; 22 ; 23 ; 27$ (Fig 6B)

$\checkmark$ Puente fijo provisional: 15-17

$\checkmark$ Prótesis parcial removible acrílica (Wipla)

\section{Maxilar inferior}

$\checkmark$ Corona provisional pzas. 31;32;33;34;35;36;37;41;42;43;44,45,46,47 (Fig 6B)

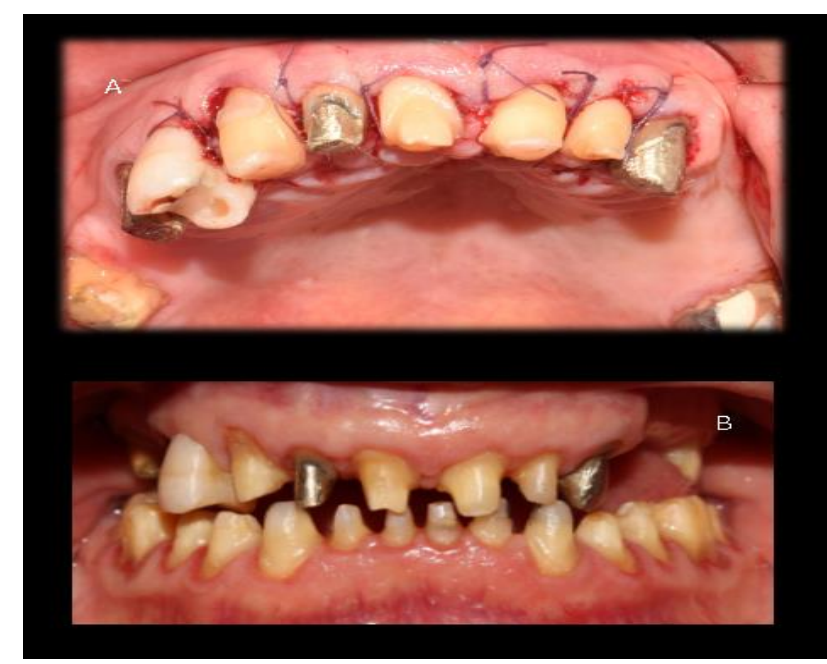

Fig. 6: Tallados de las piezas y pernos instalados.

\section{Fase definitiva:}

Maxilar superior

$\checkmark \quad$ Restauración con resina: 13,14 (v) ;24 (o)

$\checkmark$ Poste colado: 12;15;21;23

$\checkmark$ Coronas metal- cerámicas pzas: $11 ; 12 ; 13 ; 14 ; 21 ; 22 ; 23 ; 27$ (fig 8 a,b,c)

$\checkmark$ Puente metal-cerámico pzas :15-17 (Fig 8 a, b, c)

$\checkmark$ PPR Cr-Co

$\checkmark$ Férula miorelajante
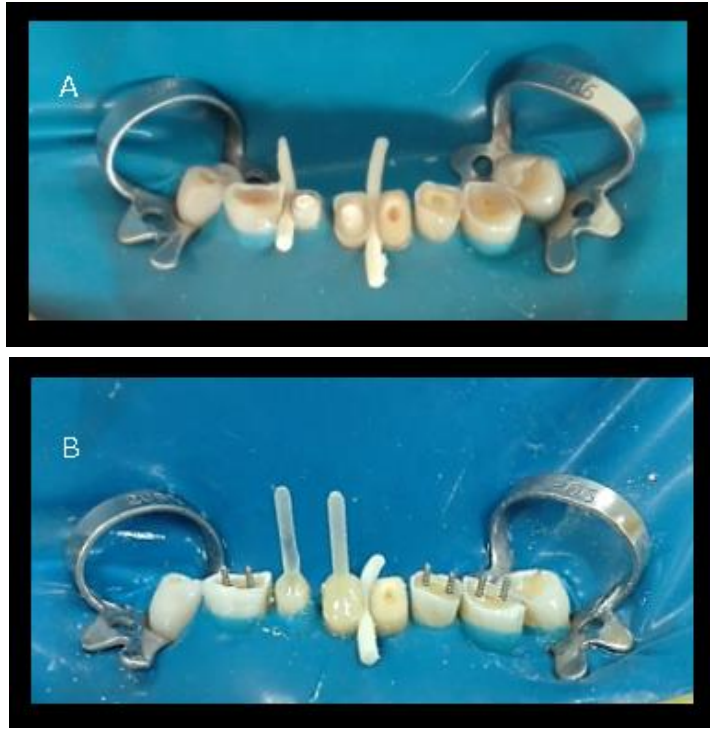

Fig. 7: Preparación de los conductos Inferiores

\section{Maxilar Inferior}

$\checkmark$ Poste de fibra pza 31;41;42 (Fig 7 a, b)

$\checkmark$ Reconstrucción con resina y pines dentinarios $31 ; 32 ; 33 ; 41 ; 42 ; 43$ (Fig 7 a, b)

$\checkmark$ Coronas metal-cerámicas pzas. $31 ; 32 ; 33 ; 34 ; 35 ; 36 ; 37 ; 41 ; 42 ; 43 ; 44 ; 45,46,47(\mathrm{Fi}$ g. 8 a, b, c)

Después de dos meses de estabilizado con coronas y puentes provisionales aumentando la dimensión vertical y verificando el perfil acompañado del confort del paciente se procedió a realizar la fase definitiva con las respectivas verificaciones en cada uno de los pasos: prueba de cofia de metal, de porcelana en movimientos céntrico y excéntricos haciéndose los ajustes correspondientes en cada una de ellas. (Fig 9 a, b, c, d,e,f); (Fig 10 a,b,c,d,e);(Fig 11)

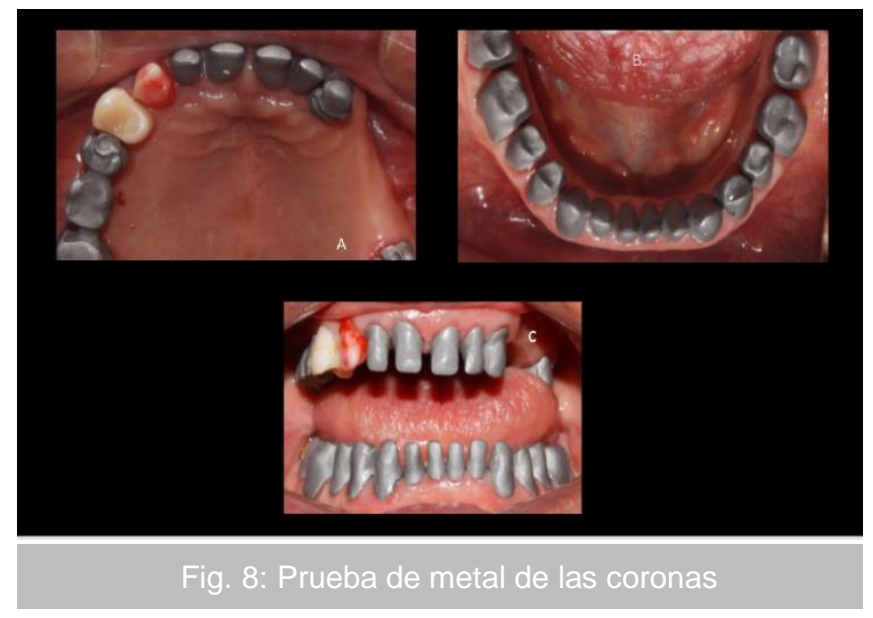




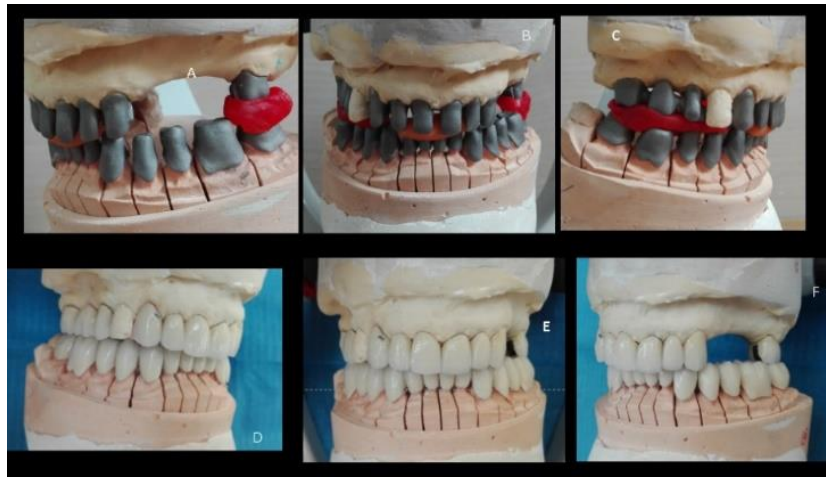

Fig. 9 Prueba de metal y prueba de corona definitivas

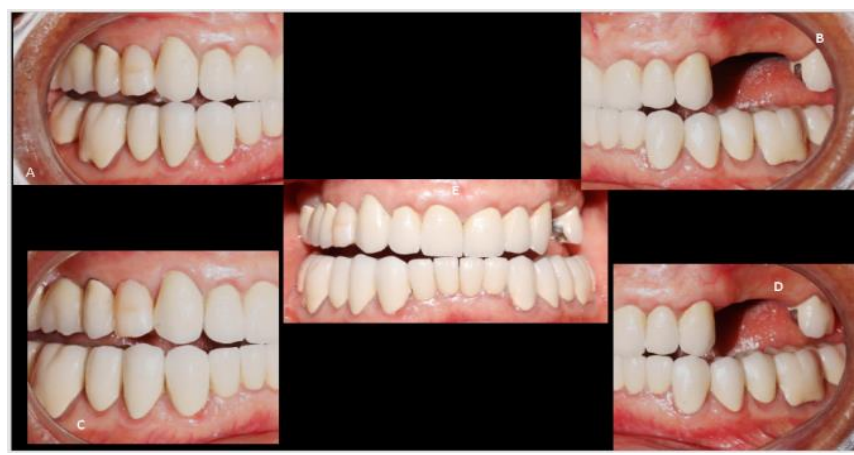

Fig. 10 Coronas instaladas

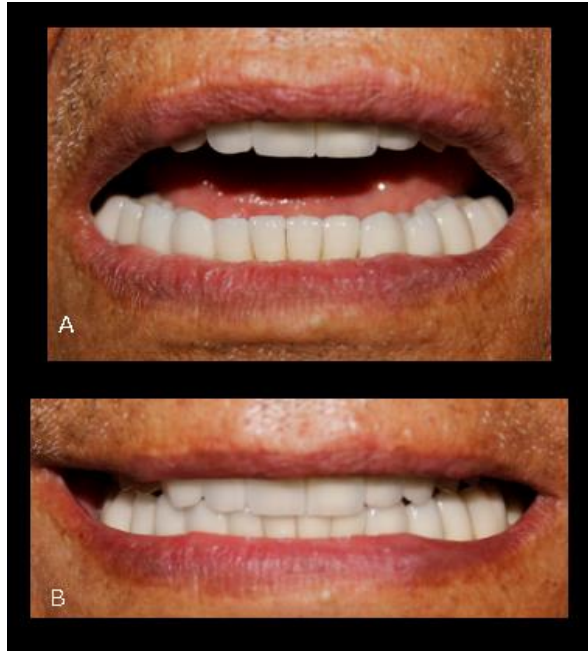

Fig. 11 En apertura y cierre con las coronas definitivas.

Después de comprobar la oclusión adecuada en el enfilado (Fig. 12 a, b, c), se procedió a realizar el acrilizado y posteriormente la instalación de la PPR con doble eje (Fig. 13).

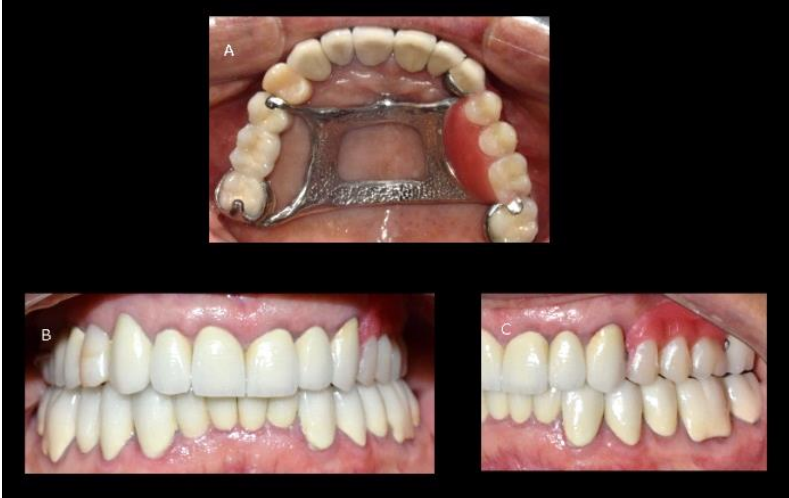

Fig. 12 Prueba de PPR metálica con enfilado.

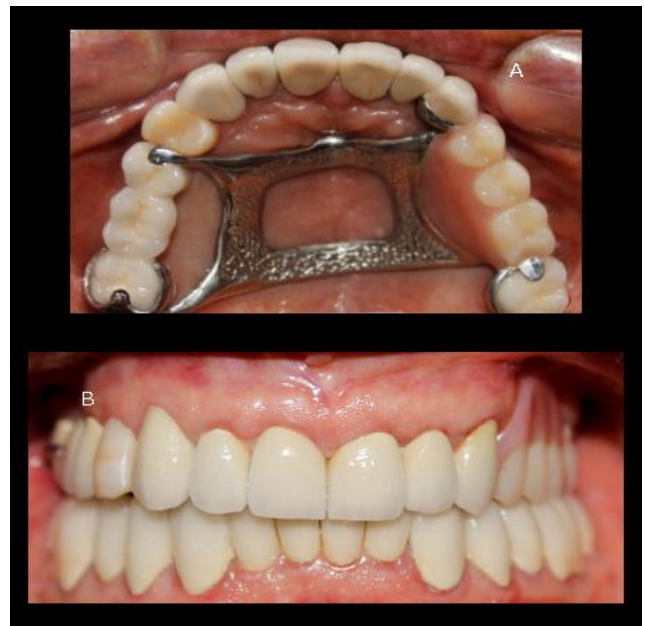

Fig. 13 PPR de doble eje terminada $€$ instalade

Se realizaron los controles posteriores a la instalación y se procedió a la confección de una férula de protección. Terminadas las evaluaciones correspondientes se dio de alta al paciente. (Fig. $14 \mathrm{a}, \mathrm{b}$ ).

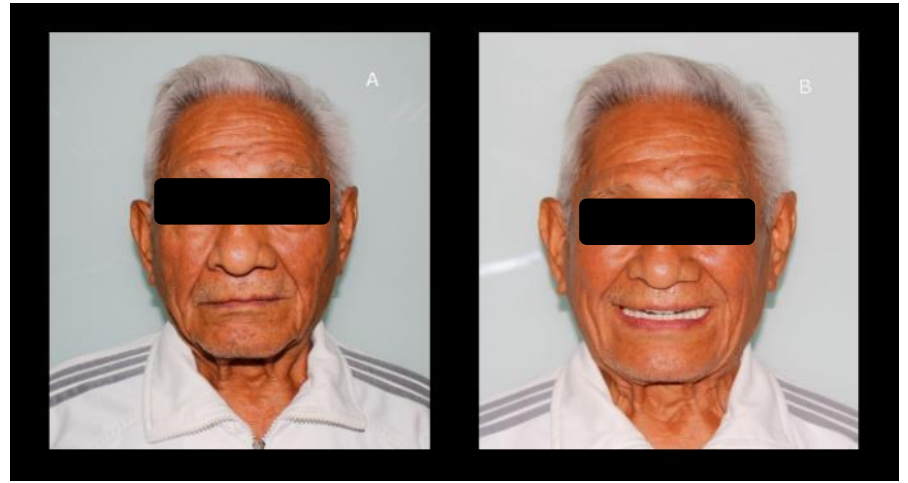

Fig. 14: Fotos faciales del paciente con la rehabilitación instalada en boca. 


\section{DISCUSIÓN}

La prótesis combinada fija y removible resulta ser una alternativa en aquellos pacientes que no puedan por diversos motivos, acceder a prótesis sofisticadas 0 tratamientos implantológicos ${ }^{(3-5)}$. En este caso clínico se cumplió con todos los parámetros necesarios para establecer una recuperación de la dimensión vertical en primera instancia con coronas provisionales y prótesis acrílica, posteriormente ya estable el paciente se hizo el cambio por PPF metal cerámica y PPR Cr-Co de doble eje de inserción, los cuales nos permitieron mantener una adecuada función del sistema estomatognático. En cuanto a la parte estética, también se pudo lograr el objetivo, consiguiendo uniformidad de características entre los dientes artificiales fijos y removibles, logrando así el alta del tratamiento debido a que se devolvió función y estética la paciente.

Según el procedimiento realizado, podemos decir que el manejo clínico de la DV, es decir cualquier modificación a realizar debe considerar: en primer lugar, el punto de partida para la reconstrucción de la dimensión vertical en oclusión debe ser en posición de relación céntrica.6 Una vez establecido el aumento de la dimensión vertical es importante también certificar que la restauración de esta medida de aumento es la adecuada, esto se realiza a través de la adaptación neuromuscular que se puede comprobar por el confort del paciente y la ausencia de sintomatología. ${ }^{7,8}$.Se recomienda manejar la DV con provisionales, a los cuales se les denomina testigos de la DV, porque permiten al paciente adaptarse a una nueva DV, los cuales posteriormente nos permiten realizar la transferencia de esa posición a los definitivos ${ }^{(9-11)}$. En cuanto a las prótesis removibles pueden tener calidad estética mejorada, si sus bases acrílicas reciben caracterización y se realizan copiando las semejanzas del tejido gingival. La caracterización de las bases favorece la aceptación de las prótesis por parte de los pacientes. (12)

\section{CONCLUSIONES}

- La prótesis parcial removible es una alternativa para el tratamiento del edéntulo parcial.

- La atención a los detalles en todas las etapas del tratamiento (puede) asegura una rehabilitación protésica exitosa.

- Es ventajoso combinar restauraciones individuales de cobertura total tipo metal cerámica, con prótesis parcial removible, cuando existen migraciones y malposiciones moderadas de los pilares.

- Se logra restaurar la DVO disminuida con prótesis combinadas fija y removible.

- Se consigue el funcionamiento armonioso y fisiológico de las prótesis combinadas.

- Es necesario educar al paciente sobre la importancia de un buen mantenimiento de los elementos protésicos y de las estructuras bucales residuales.

\section{REFERENCIAS BIBLIOGRÁFICAS}

1. Dawson P. Oclusiòn funcional: diseño de la sonrisa a partir del ATM. Caracas: Amolca. 2009.

2.Lauritzen, G. Atlas de análisis oclusal. Traductor Agustín Campos Ortega. H.F. Primera edición española. Martínez de Murguía Editores. Madrid 1977. XVII + 255 pp.

3. Mallat Desplats, E. "Prótesis parcial removible", Ed. Harcourt Brace de España. 1ํ impresión 1998

4.Pegoraro IF. "Prótesis Fija" Edit.Artes Médicas Latinoamérica, 1edición, Sao Paulo 2001.

5.Castillo A. Manejo clínico de la dimensión vertical con prótesis mixta: Reporte de caso clínico.[Monografía de especialidad en Rehabilitación Oral]. Lima - Perú: Escuela de Post-Grado, Universidad Nacional Mayor de San Marcos; 2009

6.Pairazaman Juan. Recuperación de la dimensión vertical con prótesis combinada: reporte de caso clínico rev. salud \& vida sipanense. 2016; (1):61 - 74

7.Loza D, Rodney H. Diseño de Prótesis Parcial Removible. 1era ed. Madrid: Editorial Ripano S.A. 2006.

8.Mezzomo E. "Rehabilitación oral para el clínico" Edit. Amolca 2003.

9.Colombo R, Delgado V. Manejo clínico de la dimensión Vertical. Gaceta dental.2006; 175: 94-102.

10.Matta C, Sagawa J, Comparación entre la zona facial media y el tercio facial inferior en estudiantes de 19 a 25 años de edad de la Facultad de Estomatología de la UPCH. Revista Estomatológica Herediana. 2003; 13(2):12

11. Harper R. Indicaciones clínicas para modificar la dimensión vertical en oclusión, consideraciones funcionales y biológicas para la reconstrucción de la oclusión dentaria. Quintessence Internacional. 2000; 31 (4).

12. Colmenares N. Sanchez A. Prótesis parcial removible con eje de inserción rotacional [tesis postgrado]. Venezuela: universidad central de Venezuela; 2003 